Vol. 12, $n^{\circ} 1 \mid 2008$

Varia

\title{
Bertillon, l'anthropologie criminelle et l'histoire naturelle : des réponses au brouillage des identités
}

Dominique Guillo

\section{(2) OpenEdition \\ Journals}

Édition électronique

URL : https://journals.openedition.org/chs/70

DOI : $10.4000 /$ chs. 70

ISSN : 1663-4837

Éditeur

Librairie Droz

\section{Édition imprimée}

Date de publication : 1 avril 2008

Pagination : 97-117

ISBN : 978-2-600-01237-9

ISSN : $1422-0857$

\section{Référence électronique}

Dominique Guillo, «Bertillon, l'anthropologie criminelle et l'histoire naturelle : des réponses au brouillage des identités », Crime, Histoire \& Sociétés / Crime, History \& Societies [En ligne], Vol. 12, n 1 ] 2008, mis en ligne le 01 avril 2008, consulté le 24 mars 2022. URL : http://journals.openedition.org/ chs/70 ; DOl : https://doi.org/10.4000/chs.70

Ce document a été généré automatiquement le 24 mars 2022.

(c) Droz 


\title{
Bertillon, l'anthropologie criminelle et l'histoire naturelle : des réponses au brouillage des identités
}

\author{
Dominique Guillo
}

1 Dans les débats qui se nouent en France à la fin du dix-neuvième siècle autour de la criminalité, la référence à la biologie, plus précisément à l'histoire naturelle ${ }^{2}$, revient avec insistance. Policiers, représentants des administrations judiciaires, juristes, médecins, statisticiens ou anthropologues, nombre de ceux qui traitent d'une manière ou d'une autre du crime, invoquent la nécessité de prêter une attention toute particulière au corps des criminels. Dans les commentaires qui lui sont aujourd'hui consacrés, cet intérêt pour le corps est fréquemment présenté comme l'expression d'un même fonds discursif, assez semblable chez les différents acteurs. Les discours des uns et des autres sont ainsi décrits comme autant d'actualisations d'une idéologie «biologisante », visant à présenter comme fondés en nature les hiérarchies sociales et les critères de la normalité propres aux classes dominantes. Ainsi insiste-t-on dans ces commentaires sur les éléments par lesquels les différents acteurs prétendent mettre au jour les fondements organiques et innés du crime, autrement dit sur le registre causal, réductionniste et essentialiste de ces discours ${ }^{3}$.

2 Pour importante qu'elle soit, cette dimension de l'usage de la biologie ne doit pas masquer d'autres aspects essentiels de cet intérêt porté au corps et à l'histoire naturelle, moins fréquemment soulignés, quoique fort importants. En effet, ces discours ne se situent pas uniquement dans le registre causal du fondement organique des dispositions à commettre des crimes. Ils constituent également autant de constructions intellectuelles destinées à réordonner les identités individuelles et collectives, considérablement brouillées durant cette période. En un mot, ces discours se déploient, également, dans le registre indiciaire des signes de l'identité. Et, à ce titre, ils n'ont pas simplement vocation à naturaliser les hiérarchies et les normes sociales nouvelles en assignant aux conduites que celles-ci prohibent une cause logée dans le corps, mais, également, à répondre au problème de l'identité et de l'identification, tel 
qu'il se pose en cette fin de siècle. Autant que de causes biologiques du crime, il est donc ici question d'indices de l'identité individuelle ou de l'appartenance à une identité collective ${ }^{4}$.

3 L'objectif du présent article est de suggérer que l'on peut tirer des enseignements originaux en explorant ce second aspect de ces discours, à travers l'étude comparée de deux sources: la référence que fait Alphonse Bertillon à l'histoire naturelle pour identifier les criminels récidivistes, d'une part, et l'usage que font de cette même histoire naturelle une partie des anthropologues qui s'intéressent au crime, d'autre part 5 .

4 La perspective que l'on propose permet de montrer, tout d'abord, à la différence de l'interprétation fondée sur l'hypothèse de l'empire d'une idéologie biologisante essentialiste sur les discours, que les logiques qui sous-tendent l'utilisation de l'histoire naturelle peuvent être, en réalité, fort différentes chez les acteurs évoqués supra, même si l'on peut observer des convergences et des similitudes dans les arguments, les références générales ou le lexique employés. Ainsi Bertillon se sert-il de l'histoire naturelle à des fins et sous des modalités bien distinctes de celles des anthropologues criminologistes: il tire parti des travaux de l'histoire naturelle consacrés à la reconnaissance de l'identité individuelle; les seconds, quant à eux, mettent à profit les principes élaborés par les naturalistes pour obtenir une connaissance des identités génériques des êtres vivants, deux entreprises dont la différence est perçue alors avec de plus en plus d'acuité dans les sciences de la vie.

5 Ensuite, sous l'éclairage de la perspective développée ici, ces deux usages attirent l'attention, chacun d'une façon bien spécifique, sur un fait sans doute capital pour expliquer l'intérêt porté au corps du criminel dans les dernières décennies du dixneuvième siècle. Cet intérêt pourrait tenir, pour partie, au brouillage qui affecte alors l'identification individuelle et générique d'autrui, dans une société qui est de moins en moins fondée sur une « logique du face-à-face " ${ }^{6}$, où la foule anonyme devient peu à peu l'expérience quotidienne. Un tel constat suggère que les discours qui invoquent les sciences de la vie à propos du crime au dix-neuvième siècle ont d'importantes spécificités conceptuelles et contextuelles par lesquelles ils se distinguent des discours biologisants actuels sur le crime.

\section{Le problème de la récidive}

6 L'usage que fait Bertillon de l'histoire naturelle obéit à un objectif bien précis : résoudre le problème de la récidive. Comme l'a montré Bernard Schnapper, l'« obsession de la récidive » (Schnapper, 1983) hante tout au long du dix-neuvième siècle les discours et les pratiques visant à corriger le crime. Le "criminel endurci» (voir Briegel, Porret, 2006) est de plus en plus regardé comme un individu bien spécifique, qui doit être nettement distingué du criminel d'occasion - ou primaire--, lequel est considéré comme amendable, du moins en principe, et susceptible d'être remis dans le droit chemin par de saines influences.

7 Cette obsession se traduit au plan juridique par des lois, en particulier, pour la période qui nous occupe, la loi du 27 mai 1885 sur la relégation des récidivistes. Cette loi, et plus largement, le souci qu'elle exprime dans les institutions qui ont pour but de traiter le crime, impliquent que l'identification devient «la condition élémentaire de la répression » (Kaluszynski, 2002, p. 140, citée in About, 2004, p. 30). En effet, pour cerner 
la population des récidivistes, il faut connaitre la carrière criminelle des individus aux prises avec les institutions judiciaires, savoir lesquels ont des antécédents en la matière. Et ceci suppose que l'institution soit capable d'avoir une certitude sur l'identité des prévenus, qu'elle garde en mémoire les renseignements qu'elle a pu accumuler à leur propos, et qu'elle les mette rapidement à la disposition de ceux qui cherchent à déterminer sans erreur possible une identité .

De telles exigences rejaillissent donc nécessairement sur l'orientation du travail de la police, qui doit accorder une place centrale à la détermination de l'identité. Ainsi, dans les premières décennies de la Troisième République, l'identification des récidivistes devient l'un de ses objectifs majeurs ${ }^{8}$. Et sur cette question, les policiers ont le sentiment que cet objectif ne saurait être rempli de façon satisfaisante sans une modification profonde des procédures d'identification. En effet, la récidive est perçue comme la conséquence directe des facilités offertes à la dissimulation de l'identité et, corrélativement, des difficultés pour les services d'identification judiciaire à retrouver un suspect sur le territoire, ou à découvrir l'identité réelle d'un individu lors d'une arrestation. Il est enfantin, déplore-t-on alors, de duper le policier, le juge ou le gardien de prison. Perversité ultime d'un tel état des choses, certains grands criminels, pour ainsi dire, se cachent à l'intérieur même des prisons, en purgeant une peine pour délit mineur sous un faux nom ${ }^{9}$.

Les recherches menées sur cette question ont permis d'établir que jusqu'aux années 1880, la police française utilisait deux procédés administratifs principaux pour reconnaître l'identité de ces criminels patentés ${ }^{10}$ : les fiches de signalement et les photographies des individus conduits au dépôt ${ }^{11}$. Or, ces deux moyens étaient inefficaces. Il fallait, en effet, comparer systématiquement chaque prévenu avec des milliers de fiches et de photographies, et, chaque fois, prêter une attention extrême à la modification des traits ou au vieillissement. Outre sa lourdeur administrative, une telle méthode reposait sur l'appréciation d'une ressemblance visuelle, dont les résultats, de fait, demeuraient très insatisfaisants et ne permettaient guère de repérer le récidiviste. En définitive, de telles procédures échouaient souvent à atteindre l'identité réelle d'un inconnu ou d'un imposteur, parce qu'elles consistaient à comparer un individu singulier avec des milliers de signalements singuliers, parce qu'elles reposaient tout entières sur l'improbable possibilité de retrouver une trace, un sceau original donnés l'aspect de la personne arrêtée - parmi cette multitude de cachets idiosyncrasiques que constituait l'empilement, sans ordre possible, des fiches et des photographies ${ }^{12}$.

10 Tels furent, d'ailleurs, dans la dernière décennie du siècle, les motifs de quelques réticences initiales envers l'usage des empreintes digitales dans l'identification judiciaire, prôné alors en particulier par Francis Galton ${ }^{13}$. Certes, comme la photographie, elles pouvaient permettre de reconnaitre un coupable lorsque le nombre des suspects était limité. Mais elles restaient également impuissantes, faisait-on valoir, à résoudre le problème crucial de l'identification des criminels cachés sous un faux nom et, plus généralement, celui de l'identification administrative.

11 C'est en s'inspirant de l'histoire naturelle qu'Alphonse Bertillon propose dans les années 1880 une méthode, rapidement fameuse, destinée à résoudre toutes ces difficultés et à assurer la reconnaissance des récidivistes. 


\section{La « méthode anthropométrique »}

12 Fils du célèbre médecin, anthropologue et statisticien Louis-Adolphe Bertillon ${ }^{14}$, Alphonse Bertillon entre en 1879, après des études peu brillantes, sur recommandation de son père, à la police municipale de Paris comme modeste commis auxiliaire aux écritures. Chargé de la rédaction des fiches de signalement, le jeune homme est bientôt frappé par la profonde imperfection d'un tel procédé d'identification. Ayant hérité de son père la passion de l'anthropométrie et des statistiques, Bertillon imagine alors, comme l'on sait, une méthode inspirée de l'histoire naturelle - l'«identification anthropométrique $»^{15}$ - qui, après quelques déboires, est acceptée par le préfet Camescasse en 1880. De spectaculaires arrestations, comme celle de Ravachol en 1892, identifié sous les traits d'un certain Kœnigstein emprisonné à Saint-Étienne deux ans auparavant, assurent bientôt une renommée internationale à celui que beaucoup considèrent alors comme le Pasteur de l'ordre social, dont la "police scientifique " débarrassera bientôt la société de ses « microbes $»^{16}$.

13 La méthode mise au point par Bertillon part d'une réflexion sur la nature de l'activité policière. "À regarder les choses d'un point de vue élevé ", souligne-t-il dans un ouvrage destiné à donner les instructions relatives à l'application de son procédé, «tout en police est affaire d'identification». Ce qui incombe, en définitive, au policier, c'est d'« individualiser» le criminel « au milieu de la foule des humains. [...]. Depuis le commencement jusqu'à la fin de l'enquête judiciaire, ce ne sont que des questions d'identité, de description, de signalement à élucider » (Bertillon, 1893, p. VI, souligné par Bertillon) ${ }^{17}$.

14 Or, aux yeux de Bertillon, un tel objectif rapproche l'activité policière de l'anthropologie. Celle-là, comme celle-ci, cherche à identifier, à reconnaître des corps individuels :

15 "L'anthropologie, dit-il ainsi, n'est pas autre chose, par définition, que l'histoire naturelle de l'homme. Est-ce que de tous temps les chasseurs ne se sont pas piqués de connaissances en histoire naturelle et inversement, les naturalistes ne sont-ils pas d'instinct un peu chasseurs? Nul doute que les policiers de l'avenir n'arrivent à appliquer à leur chasse particulière les règles de l'anthropologie, tout comme les chauffeurs de nos locomotives mettent en pratique les lois de la mécanique et de la thermodynamie » (Bertillon, 1893, p. VII) ${ }^{18}$.

16 Aussi est-il permis, selon Bertillon, d'espérer soustraire la recherche des criminels aux errements de l'intuition et de la routine en empruntant à la science du corps humain les éléments d'une méthode véritablement scientifique. Cette anthropologie, ou histoire naturelle de l'homme, livre, selon lui, les principes à suivre pour obtenir des critères de « signalement » efficaces : les critères « anthropométriques ».

17 En effet, souligne Bertillon, trois faits établis par l'anthropologie sont à considérer avec attention : «la fixité à peu près absolue de l'ossature humaine à partir de la vingtième année d'âge "; "la diversité extrême de dimension que présente le squelette humain comparé d'un sujet à un autre »; et «la facilité et la précision relative avec lesquelles certaines dimensions du squelette sont susceptibles d'être mesurées » (Bertillon, 1893, p. XVI).

18 De ces trois faits, ajoute-t-il, dérive une conséquence capitale: pour peu que l'on choisisse un trait physique facilement mesurable et dont les dimensions varient 
sensiblement d'un homme à l'autre - la taille par exemple -, il est possible d'obtenir une classification stable de n'importe quelle population humaine en plusieurs groupes significatifs d'effectifs égaux, en divisant en autant de classes que l'on veut le continuum des valeurs prises par ce critère. Si l'on subdivise ensuite chacun des groupes obtenus précédemment en classes d'effectif égal au moyen d'un autre critère la longueur du pied gauche, par exemple-, on obtient, dans chacun des groupes initiaux, des sous-groupes plus précis, qui renferment un nombre plus faible d'individus combinant deux propriétés - par exemple, les personnes dont la taille est comprise entre un $1 \mathrm{~m} 65$ et $1 \mathrm{~m} 68$ et dont le pied gauche mesure entre $25 \mathrm{~cm}$ et $25,5 \mathrm{~cm}$. En répétant à nouveau le même procédé, on finit par obtenir une distribution de la population en classes dont les effectifs sont très restreints - on peut aller ainsi jusqu'au singleton - et dont les éléments ont en commun autant de propriétés métriques qu'il y a de rangs de subdivision. "On l'a dit depuis longtemps, rappelle Bertillon, qu'il était impossible de trouver deux feuilles semblables : jamais la nature ne se répète " (Bertillon, 1893, p. XV). Il est donc toujours possible de resserrer un peu plus l'étau des intervalles de variation des mensurations avec une nouvelle division pour distinguer deux individus.

Pour peu que l'on effectue des mesures appropriées sur toutes les personnes qui passent entre les mains de la police, ajoute ensuite Bertillon, et qu'on les classe selon cette méthode, on obtient un classement de fichiers qui doit permettre, en principe, de déceler le récidiviste, tout comme le naturaliste, armé de ses tables dichotomiques, parvient à identifier les plantes ou les animaux singuliers qu'il rencontre dans la nature. Pour déterminer ou vérifier l'identité d'un suspect, on commencera par mesurer, sur lui, la taille de la partie du corps au moyen de laquelle est effectué le premier rang de subdivision dans le fichier général. Avec le chiffre obtenu, on saura que l'identité de l'individu se trouve dans un groupe, ou, si l'on veut, un fichier particulier bien précis - s'il a déjà eu affaire à la police. Ensuite, on procèdera à la mesure de l'organe correspondant au second rang taxinomique, puis, en fonction du chiffre obtenu, on retiendra la subdivision du premier groupe correspondant à la nouvelle mesure. Et ainsi de suite, jusqu'à parvenir à un fichier comprenant suffisamment peu d'individus pour que les autres procédés d'identification empreintes, photographie, etc. - puissent donner des résultats probants.

Point essentiel, pour donner un maximum d'efficacité à un tel dispositif classificatoire, il faut ordonner les critères - les «signalements » - selon leur capacité à différencier les individus, c'est-à-dire selon leur pouvoir de discrimination :

21 "L'ordre de la classification [des critères] est déterminé d'après la considération [suivante] : placer au début les mesures qui ont la plus grande puissance signalétique, c'est-à-dire celles qui sont à la fois les plus stables chez le même individu et les plus variables d'un individu à un autre, de façon que les éliminations effectuées en premier prêtent le moins possible aux hésitations et aux recherches doubles qui en découlent. Ces dernières sont en effet d'autant plus rapidement effectuées qu'elles portent sur les ramifications ultimes de la classification" (Bertillon, 1893, p.XXIV, c'est nous qui soulignons) $)^{19}$.

D'après les observations de Bertillon, la taille est, de toutes les variables anthropométriques, celle qui a la plus grande "puissance signalétique » ou "valeur récognitive " (Bertillon, 1893, p. XXIX, souligné par Bertillon). Suivent, dans l'ordre, l'« envergure ", la « hauteur du buste », la "longueur de la tête», sa «largeur », la 
«longueur de l'oreille droite », la « largeur de l'oreille droite », la «longueur du pied gauche », la "longueur du médius gauche », la «longueur de l'auriculaire gauche » et, enfin, la « longueur de la coudée gauche » (Bertillon, 1893, p. XXVI). Tels sont, au total, selon Bertillon, les critères du dispositif taxinomique le plus efficace pour individualiser les corps et débusquer l'identité individuelle.

\section{Méthode anthropométrique et anthropologie criminelle : deux usages de l'histoire naturelle}

Bien que les références à l'histoire naturelle, à l'anthropologie et aux théories statistiques de son temps reviennent fréquemment sous la plume de Bertillon, la méthode d'identification qu'il propose doit peu, en réalité, aux thèses spécifiques développées dans le cadre de ces disciplines. Plus encore, sur deux points importants, la méthode de Bertillon est contradictoire avec la théorie de l'«homme moyen" de Quetelet et avec celle de l'« homme criminel » des criminologistes italiens et d'une partie des anthropologues naturalistes.

Premier point, l'existence d'un « homme » ou d'un type criminels et, plus généralement, de types physiques bien distincts, implique que les formes des différentes parties d'un corps soient corrélées les unes aux autres, varient conjointement dans la population étudiée : telle taille ou forme d'oreille ira avec telle taille ou forme de nez, etc. Or si l'on retient une interprétation radicale de cette hypothèse, elle implique l'impossibilité de progresser dans la discrimination des individus au moyen de subdivisions dans la population de départ : la taille, par exemple, sera corrélée à l'envergure, qui elle-même sera dans un rapport stable avec la longueur de tête, etc. Ainsi, s'il existait des types parfaits en leur genre d'êtres humains, en particulier un type criminel, l'anthropologue - ou le policier - obtiendrait les mêmes collections d'individus, les mêmes "espèces", du premier au dernier rang de la classification. Les criminels auraient un corps semblable; et le policier se retrouverait face à l'homogénéité du «type criminel », qui interdirait la possibilité de subdiviser son fichier de départ.

Bien entendu, l'idée de "type " ou, si l'on veut, de corrélation, de dépendance réciproque des traits organiques propres à un groupe d'individus, n'est jamais employée en un sens aussi radical par les anthropologues criminologistes comme par les naturalistes. Aussi la méthode de Bertillon peut-elle, en pratique, cohabiter sans trop de difficultés avec les théories du «type criminel ». Mais il y a bien, sur ce point, une tension entre les principes fondamentaux qui sous-tendent les descriptions du corps criminel livrées respectivement par Bertillon, d'une part, et par les anthropologues criminologistes, d'autre part. Les caractères organiques retenus par Bertillon doivent avoir un degré de corrélation minimum - sans quoi la méthode n'est pas efficace, puisqu'elle élimine moins d'identités possibles à chaque nouvelle mesure effectuée, à chaque nouveau rang de la classification des groupes d'individus; tandis que les caractères organiques choisis par les anthropologues doivent être, à l'inverse, corrélés les uns aux autres le plus possible - sans quoi on ne pourra parler de l'existence d'un type morphologique du criminel.

Deuxièmement, les caractères organiques que recherche Bertillon ont une autre propriété antinomique avec celles des caractères que font valoir la statistique criminelle appuyée sur le concept d' " homme moyen ", d'une part, et l'anthropologie à la recherche d'un type criminel, d'autre part. En effet, ces deux disciplines s'appuient 
largement sur l'association étroite des concepts de normalité et de moyenne. Dans un tel cadre, les criminels sont regardés comme des " marginaux » ou des "déviants » au sens de la statistique (voir Desrosières, 1993). Et les caractères susceptibles d'étayer de telles hypothèses doivent avoir des distributions «normales » ou gaussiennes, centrées sur la moyenne, c'est-à-dire en cloche. Or, chez Bertillon, le caractère organique retenu pour le fichier anthropométrique doit avoir, à l'inverse, la distribution la moins «normale» et la plus «plate» possible. Bien entendu, il ne s'agit pas de nier, pour Bertillon, que la plupart des caractéristiques physiques ont généralement une distribution «normale" dans les populations humaines. Simplement, pour être efficace, la méthode de Bertillon implique, comme il le dit lui-même, que l'on choisisse des caractères dont les valeurs « varient » le plus possible d'un individu à l'autre. Car plus les valeurs d'un caractère physique varient dans une population, moins elles sont resserrées autour de la moyenne - leur écart-type est plus important - et plus il est facile, en conséquence, d'obtenir un nombre important de groupes d'effectif égal délimités par des intervalles de valeurs suffisamment grands pour ne pas avoir de doute dans le classement des mesures effectuées. En effet, si les intervalles de valeurs que l'on retient sont trop étroits - ce à quoi l'on est condamné lorsque la plupart des individus sont proches des mêmes valeurs pour un caractère donné dans une population - le degré de précision exigé dans la mesure pour classer les individus avec certitude est si grand qu'il est difficile à obtenir. En résumé, la qualité, la « puissance signalétique » d'un caractère anthropométrique est inversement proportionnelle à sa « normalité » statistique. Pour cette raison, la méthode anthropométrique ne peut être simplement regardée comme une des actualisations ou un des avatars de l'idéologie de la norme comme moyenne et de la déviance comme marginalité statistique, qui imprègne alors une partie de la statistique criminelle et de l'anthropologie ${ }^{20}$.

Reste que les anthropologues, tout comme Bertillon, s'appuient très explicitement sur l'histoire naturelle. En effet, l'anthropologie criminelle se développe tout au long de la seconde moitié du dix-neuvième siècle à partir des liens intimes qui se tissent entre, d'un part, l'anthropologie naissante, œuvre de naturalistes, souvent également médecins, qui entendent mener à bien le projet d'une « histoire naturelle de l'homme " caressé par Buffon (voir Blanckaert, 1989), et, d'autre part, une partie de la médecine, dont certains représentants s'intéressent de très près au crime, perçu comme une pathologie inscrite dans l'« organisation » de l'individu, c'est-à-dire dans son corps en tant qu'il a été façonné par la « nature » et par l'hérédité.

Les aspects de cette histoire ont été étudiés dans le détail et sont aujourd'hui bien connus (voir en particulier Pick, 1989 ; Coffin, 1993 ; Renneville, 1997). Ainsi a-t-on pu établir que les auteurs les plus célèbres de ce courant - par exemple, Cesare Lombroso qui connaît un vif succès au moment où Bertillon développe ses premières méthodes d'identification, s'appuient sur une littérature médicale qui fleurit dès le milieu du dixneuvième siècle. On peut citer, à cet égard, les écrits de Prosper Lucas (notamment son Traité philosophique et physiologique de l'hérédité naturelle dans les états de santé et de maladie du système nerveux, publié en 1847-1850), de James Cowles Prichard et, surtout, le Traité des dégénérescences physiques, intellectuelles et morales de l'espèce humaine de BénédictAugustin Morel (1857) et La psychologie morbide dans ses rapports avec la philosophie de l'histoire de Jacques-Joseph Moreau de Tours (1859), qui contribuent à populariser dans le monde médical et, plus largement, dans le monde savant, les thèmes de la "folie morale ", de la «dégénérescence » organique du criminel, de l'« hérédité » des 
prédispositions "morbides", en un mot, de l'inscription dans le corps même du criminel de la disposition, jugée pathologique, à dévier de la norme.

Or, tout au long de la seconde moitié du siècle, ces thématiques s'associent étroitement au langage et aux questionnements de l'histoire naturelle. Comme on l'a dit, nombre de naturalistes, en particulier ceux qui s'intéressent à l'homme, sont également médecins. Et, d'autre part, dans les réflexions médicales qu'il suscite alors, le crime, parce qu'il est censé s'expliquer par le type d'«organisation" anatomique propre à celui qui le commet, est conceptualisé au moyen d'une grille lexicale qui est souvent celle de l'histoire naturelle du milieu du siècle ${ }^{21}$, c'est-à-dire une histoire naturelle appuyée sur l'anatomie comparée largement hantée, et plus encore lorsqu'il s'agit de l'homme, par le thème de l'échelle des êtres (Daudin, 1926-1927 ; Balan, 1979 ; Blanckaert, 1989, 1994 ; Guillo, 2003). Dans l'anthropologie dont s'inspire cette criminologie naturaliste, on retrouve ainsi, appliqués à l'homme, les concepts et les outils théoriques élaborés pour conceptualiser la diversité des espèces vivantes depuis Georges Cuvier, Étienne Geoffroy-Saint-Hilaire, Jean-Baptiste de Lamarck ou Johann Friedrich Blumenbach. L'humanité est ainsi divisée en sous-espèces censées correspondre à des types physiques distincts. Les différentes parties du corps propre à chaque type humain - par exemple la prostituée ${ }^{22}$ ou le criminel ${ }^{23}-$, conformément au principe énoncé par Cuvier pour les animaux, sont considérées comme "corrélées» les unes aux autres: telle forme d'une partie du corps, soutient-on dans le cadre de cette anthropologie, s'accompagnera de telle autre forme définie dans les autres régions de l'organisme. En vertu de ce "principe de corrélation », la forme spécifique prise par une partie bien choisie du corps pourra servir, argumente-t-on alors, d'indice à toutes les autres. En d'autres termes, certains caractères - par exemple, pour certains anthropologues naturalistes, l'«angle facial»- pourront servir d'enseigne, de signe permettant d'indiquer l'appartenance à une nature spécifique, à un type particulier d'êtres humains. Et comme les caractéristiques physiques sont elles-mêmes regardées, dans un tel cadre, comme autant d'éléments corrélés à des caractères psychologiques et à des penchants bien définis, il devient possible de bâtir des classifications des types humains fondées sur des caractéristiques morphologiques et destinées à rendre raison des différences de mœurs et de conduites.

On peut ensuite, comme le font alors certains naturalistes avec les espèces animales, ranger les différentes sous-espèces dont l'humanité est censée se composer - les " races ", les types ou les classes - en une « série linéaire et ascendante " graduée selon le degré de complexité de l'organisation du corps et couronnée par l'homme des classes supérieures des sociétés occidentales. Dans une telle perspective naturaliste, cette "série anthropologique » fondée sur l'anatomie, dont le principe est communément accepté dans une bonne partie des discours à vocation scientifique sur l'homme tout au long du siècle ${ }^{24}$, est rattachée continûment à la "série zoologique " par l'intermédiaire des « races inférieures", dont l'organisme et les penchants sont regardés comme fort proches de ceux des singes. Enfin, ajoutant à ces vues une perspective évolutionniste, nombreux sont les anthropologues du dernier tiers du siècle qui considèrent que la série anthropologique ainsi bâtie constitue la trame du développement historique de l'humanité. Dans un tel cadre, les déviants des sociétés modernes sont fréquemment présentés comme des individus dont le corps et les penchants se rapprochent des races ou des types humains jugés frustes et primitifs, voire de certains animaux. Ils sont 
considérés comme une survivance héréditaire ou une résurgence par évolution rétrograde - par " dégénérescence »- de types primitifs dans la société moderne ${ }^{25}$.

Comme on peut en juger, l'anthropologie criminelle s'appuie donc très directement et profondément sur l'histoire naturelle pour conceptualiser le thème du type physique spécifique de l'homme criminel. Comment expliquer, dès lors, la tension qui existe entre le fonds de l'argumentaire développé par Bertillon, d'une part, et celui des criminologues naturalistes, d'autre part? Comment expliquer qu'à partir de cette même référence à l'histoire naturelle, ils parviennent respectivement à des discours dont les principes sont fort contrastés sous certains aspects?

Une telle tension souligne les limites de l'hypothèse selon laquelle ces différents discours seraient uniquement l'expression de l'empire sur les esprits d'une idéologie naturaliste biologisante, passivement acceptée et déployée par les acteurs, si différents soient-ils. Pour intéressante qu'elle soit, cette hypothèse, dans sa version radicale, ne laisse, en effet, guère de place à quelque logique autonome d'appropriation active et sélective des argumentaires de la biologie par les différents acteurs. Cette tension montre également les limites des commentaires qui réduisent ces discours au thème des causes organiques du crime, des mécanismes organiques qui sont censés engendrer le penchant criminel. Sous ces éclairages, l'élaboration d'argumentaires aux contenus si contrastés reste en effet inexplicable, sauf à considérer que ces tensions ou divergences n'ont guère de signification et que, pour les différents acteurs, les finalités idéologiques globales l'emportent de beaucoup sur la cohérence logique ou sur des objectifs plus spécifiques.

En revanche, comme on va tenter de le montrer, la perspective évoquée en introduction - perspective qui, comme on l'a dit, envisage ces discours, également, comme autant de réflexions sur les indices de l'identité, individuelle ou collective, plutôt que comme les simples actualisations diverses d'un même air du temps « biologisant » à propos de l'étiologie du crime - permet d'éclairer cette différence dans les usages de l'histoire naturelle et de faire sa part à la logique propre poursuivie par chacun des acteurs. Elle permet, en outre, de faire ressortir avec acuité un facteur susceptible d'expliquer le succès de l'histoire naturelle en France auprès du public éclairé de la fin du dix-neuvième siècle : non pas seulement l'émergence d'une société capitaliste inégalitaire hantée par le thème de la naturalisation des dispositions et des penchants; mais, aussi, le brouillage qui affecte alors l'identification ordinaire et institutionnelle d'autrui. Ce facteur contextuel permet d'expliquer les deux usages de l'histoire naturelle évoqués ici et de rendre compte de leurs différences.

\section{Connaissance et reconnaissance de l'identité dans les classifications de l'histoire naturelle}

Pour peu que l'on compare dans le détail les usages respectifs que Bertillon et les représentants de l'anthropologie criminelle font de l'histoire naturelle, et qu'on les rapporte aux débats qui animent la science de la classification des êtres vivants depuis la fin du dix-huitième siècle, on se rend compte qu'ils tirent respectivement parti, en réalité, de deux types de procédures classificatoires dont la nature et les buts sont considérés comme bien distincts par les naturalistes depuis longtemps.

Bertillon reprend les développements que l'histoire naturelle a consacrés depuis ses origines aux moyens de la reconnaissance, de la « détermination », disait-on alors plus 
volontiers, des animaux et des végétaux. L'une des préoccupations des naturalistes, au moins depuis Linné, est, en effet, entre autres, de bâtir des classifications arborescentes composées de groupes successivement inclus les uns dans les autres, destinées à identifier l'espèce à laquelle appartient un spécimen individuel de plante ou d'animal rencontré dans la nature. De telles classifications consistent en une suite de divisions successives qui permettent, à chaque rang, d'éliminer des appartenances possibles pour l'individu dont on cherche à déterminer le nom générique ou spécifique. La méthode consiste ainsi à cheminer rang après rang en examinant, à chaque rang, la forme que prend une partie définie de l'organisme du spécimen. À chaque étape, on suit le chemin qui correspond à la forme que l'on observe effectivement sur le spécimen, jusqu'à ce que l'on arrive au dernier niveau de subdivision - celui des espèces ou des variétés - qui donne son identité.

Or depuis la Flore française de Jean-Baptiste de Lamarck (1778, pp. L-LVIII) ${ }^{26}$, ce type de question et d'opération est assez nettement distingué de l'entreprise de classification des êtres vivants proprement dite, autrement dit d'une distribution arborescente des êtres vivants destinée à livrer une connaissance de leur nature, une classification qui, à chaque rang, regroupe des individus présentant entre eux plus de ressemblances qu'ils n'en ont avec les individus des groupes dont ils sont séparés.

Certes, les deux classifications utilisent un schéma logique identique : une arborescence de groupes de plus en plus restreints successivement inclus les uns dans les autres. Mais dans les secondes, celles qui ont pour objectif d'exhiber la nature des animaux et des végétaux, les traits organiques retenus pour servir de critères de subdivision des groupes - les «caractères" indicateurs - sont censés résumer, à chaque rang (embranchements, classes, ordres, familles, genres et espèces), un nombre important de ressemblances, propres aux êtres vivants de chaque groupe qu'ils permettent de distinguer. Ils sont, en ce sens, les signes d'une nature commune, parfois dérobée au regard par les altérations de surface, comme c'est le cas, par exemple, du groupe des mammifères, qui renferme à la fois les cétacés et les mammifères terrestres.

En revanche, dans les classifications destinées à identifier des individus, à les reconnaître, le choix des « caractères ", c'est-à-dire des critères de subdivision, répond à d'autres exigences. Ils doivent permettre l'identification rapide des individus par élimination. Il n'est donc absolument pas nécessaire que les subdivisions établies à chaque rang correspondent à des groupes réels, naturels, renfermant des individus d'un même type général ou particulier. Ce qui compte ici, comme le souligne Bertillon dans sa classification, c'est la "puissance signalétique » du «caractère » choisi, autrement dit sa capacité à faire des distinctions nettes et facilement repérables entre des individus (voir Bertillon, 1893, p. XXII). Or les traits corporels qui ont la plus grande " puissance signalétique » ne sont pas nécessairement ceux qui indiquent une identité générique.

39 Autre différence, plus profonde encore, dans les classifications " naturelles ", c'est-àdire dans les classifications destinées à la connaissance des êtres, il est impossible d'intervertir l'ordre des critères, qui doivent être, en outre, corrélés les uns aux autres et s'emboîter régulièrement des premiers groupes, les plus larges, jusqu'aux espèces. À l'inverse, comme on l'a évoqué supra, pour qu'un dispositif d'identification individuelle soit efficace, il faut, précisément, que les traits retenus, comme le souligne Bertillon luimême, "varient indépendamment» les uns des autres, sans quoi les subdivisions successives sont inefficaces pour éliminer à chaque étape un maximum de groupes 
auxquels l'individu n'appartient pas. En conséquence, dans une classification destinée à l'identification des individus, les critères de subdivision peuvent être intervertis sans difficulté ; ce qui n'est pas le cas dans une classification « naturelle».

Certes, durant une bonne partie du dix-huitième siècle, ces deux objectifs ne sont pas toujours distingués avec netteté par les naturalistes, dont les classifications « systématiques » arborescentes, encore imparfaites, servent indistinctement souvent à connaître et à reconnaître, à exhiber les appartenances taxinomiques naturelles et à identifier l'espèce à laquelle appartiennent les spécimens individuels rencontrés dans la nature (voir Daudin, 1926-1927). Mais comme on l'a souligné supra, dès les années 1780, en particulier sous l'impulsion de Lamarck, la nécessité de distinguer ces deux opérations est perçue et revendiquée par la majorité des spécialistes de la classification des êtres vivants.

\section{Connaître et reconnaître : le savant et le policier}

41 La différence entre les usages que Bertillon et les criminologues font respectivement de l'histoire naturelle recoupe très exactement la distinction entre ces deux opérations effectuées par les naturalistes. Les deux objectifs évoqués dans les lignes qui précèdent - reconnaissance et connaissance - correspondent, en effet, aux types de problèmes spécifiques qu'ils sont respectivement amenés à se poser dans la position qui est la leur. Bertillon se pose un problème de police: le contrôle de l'identité individuelle. La référence spécifique qu'il fait à la biologie ne doit donc rien au hasard et est ajustée à cet objectif. Loin de sacrifier passivement à l'air du temps naturaliste, il emprunte à l'histoire naturelle un outil bien précis destiné à résoudre un problème défini : identifier le récidiviste. Son but est donc de mettre au jour les signes corporels qui permettent par un jeu d'éliminations successives d'atteindre l'identité individuelle, pour mieux contrôler celle-ci. Le criminologue naturaliste, quant à lui, poursuit un autre objectif, qui renvoie à l'autre versant de l'histoire naturelle. Il s'agit pour lui de mettre au jour les caractéristiques de la nature criminelle en général, de faire œuvre non pas de police, mais de science ; non pas de contrôler des individus mais de produire un savoir à propos de leur nature. Son objectif est donc de repérer les indices corporels susceptibles de signaler l'appartenance à l'espèce des criminels. Les signes de l'identité dont il est ici question sont destinés à servir non pas à la reconnaissance de la singularité individuelle, à la détermination d'un nom propre, mais à la connaissance générique, à la mise au jour des noms communs qui désignent des types «naturels »ici le « criminel».

Ainsi, dès lors que l'on rapporte ces différents discours aux débats menés en histoire naturelle à propos de l'identité et de ses indices, autrement dit dès lors que l'on s'attache au versant par lequel ils se rapportent à un questionnement portant sur les signes, on peut percevoir avec netteté certains aspects qui leur sont essentiels et expliquer leurs spécificités respectives. Bertillon et les criminologues se posent des questions qui, formulées en termes très généraux, relèvent du thème de l'identité, en tant qu'elle est ancrée dans le corps, et des signes corporels qui permettent d'atteindre cette identité. Mais cette question de l'identité peut se décliner sur au moins deux plans bien distincts, qui renvoient à deux objectifs différents : la reconnaissance de l'identité individuelle et la connaissance des identités génériques ou, si l'on veut, collectives ${ }^{27}$. 


\section{La théorie de l'identité judiciaire implicite du bertillonnage}

43

la finalité est exclusivement l'identification des individus, il peut sans doute être lu également, à un plan qui reste implicite chez Bertillon lui-même et sans doute chez ses contemporains, comme une théorie de l'identité individuelle judiciaire. En se diffusant et en se popularisant, de telles procédures d'identification sont susceptibles de se transformer, de devenir des catégories de perception de la nature d'autrui, chez certains acteurs sociaux du moins. Sans prétendre proposer ici autre chose qu'une simple piste, on peut donc essayer de dégager sommairement la théorie de l'individualité que recèle un tel schéma anthropométrique.

Si l'on accepte de lire les propos de Bertillon de ce point de vue, il semble qu'il adopte des idées proches de celles qui se dessinent dans l'histoire naturelle du dix-huitième siècle à propos des êtres vivants ${ }^{28}$. Dans les vues qu'il développe, le corps humain est en effet conçu, a priori, comme une combinaison de traits susceptibles de varier continûment et indépendamment les uns des autres. Dans un tel cadre, il y a autant d'individus possibles - d'êtres vivants, pour l'histoire naturelle du dix-huitième siècle (voir Daudin, 1926-1927) - qu'il y a de combinaisons entre la variété continûment graduée des formes que peut prendre chacune des parties d'un corps. L'ensemble des corps possibles forme ainsi un immense espace combinatoire, un vaste tableau continu et plein. L'identité judiciaire, telle qu'elle ressort d'une telle conception du corps, est alors le résultat d'un processus d'« individualisation » progressive par discrimination, de distinction au sein de cette nappe continue d'individus possibles. Elle n'est pas une marque singulière, comme c'est le cas lorsque l'on retient les empreintes digitales. Elle est la limite de subdivisions successives et de plus en plus fines effectuées dans un vaste tableau, subdivisions stoppées lorsqu'il ne reste plus dans la case obtenue qu'une seule et unique personne. L'identité de cette personne se confond alors avec cette limite : elle est ce que ne sont pas toutes les autres ${ }^{29}$.

\section{Des tentatives pour répondre au brouillage des identités}

Lorsque l'on prend acte que ces discours se rapportent sous certains aspects essentiels à un questionnement sur les indices, il devient possible de suggérer une hypothèse sans doute moins fréquemment évoquée que d'autres pour expliquer l'intérêt porté au corps dans les discours sur l'homme criminel du dernier tiers du siècle. En effet, il est possible que cet intérêt tienne non pas seulement au fait que la référence aux sciences de la vie permet de naturaliser les déviances et, ce faisant, l'ordre social, en les présentant comme des conduites trouvant leur cause dans les corps et dans des lignées héréditaires, mais également à ce que l'histoire naturelle, telle qu'elle est alors conçue, fournit un corpus d'outils intellectuels et un lexique attractifs pour les différents acteurs qui entendent alors répondre au brouillage affectant les critères de l'identification d'autrui. Car le brouillage des identités est d'abord un problème d'ordre indiciaire - ou, si l'on veut, sémiologique : il consiste en une perturbation des signes à travers lesquels sont perçues les singularités individuelles ou les natures génériques, collectives.

Crime, Histoire \& Sociétés / Crime, History \& Societies, Vol. 12, $n^{\circ} 1 \mid 2008$ 

reconnaissance des individus - connaissent, en effet, de profondes mutations tout au long du dix-neuvième siècle. Tout d'abord, le réseau serré des interconnaissances personnelles, qui formait la trame de la vie sociale urbaine et, plus encore, rurale, se défait progressivement, rendant la détermination de l'identité individuelle de chacun, tant par les citoyens ordinaires que par les institutions, fort délicate ${ }^{30}$. Ainsi a-t-on le sentiment que les conditions sont éminemment propices à la falsification de l'identité, à l'imposture, à la duplicité, comme l'atteste la récurrence, dans les romans, des figures aux identités multiples, que l'on parvient difficilement à démasquer - Jean Valjean, Vautrin, Edmond Dantès... - ou encore les propos de ceux qui sont en charge de la police, à commencer par Bertillon lui-même. De sorte qu'à la fin du siècle, l'identité personnelle, singulière, de l'individu - son nom propre, si l'on veut - tout comme son identité générique - son appartenance sociale, telle qu'elle est perçue - ne paraissent plus pouvoir être établies, aux yeux de beaucoup, romanciers, philosophes, statisticiens ou policiers, directement ou par le seul recoupement des témoignages, des aveux et des reconnaissances individuelles ${ }^{31}$. Pour déterminer l'identité singulière d'un individu, il faut désormais recourir à d'autres méthodes. Dans de telles circonstances, l'histoire naturelle, dans les parties qu'elle a consacrées à la reconnaissance individuelle, a pu ainsi apparaître à certains acteurs comme une réponse possible aux difficultés suscitées par cette situation nouvelle ${ }^{32}$.

47 Par ailleurs, les critères de l'identité générique - de la connaissance des identités collectives des individus - se sont profondément modifiés au cours du siècle. Avec la disparition des Ordres et des Corporations, avec la mobilité sociale croissante et l'émergence de nouvelles couches sociales, la grille des catégories ordinaires utilisées pour classer les individus semble, sous la Troisième République, opaque, mouvante et relative. À l'inverse, dans la France d'Ancien Régime, la grille qui prévalait paraissait appartenir à un ordre clair, immémorial et absolu. Aux yeux des élites intellectuelles, il faut donc reconstituer une nouvelle grille, retrouver derrière le désordre apparent un ordre réel, qui distribue les types humains en groupes susceptibles d'être regardés comme objectifs, dessinant d'authentiques identités repérables par des signes infaillibles. Or, les principes mobilisés par l'histoire naturelle pour classer les animaux en groupes «naturels" permettent de dessiner un ordre hiérarchique entre des types humains qui a l'apparence de l'objectivité. En l'ancrant dans les corps, ces principes inscrivent, en effet, l'identité générique dans un objet "naturel», dont certaines parties peuvent servir d'indices visibles révélant la «nature » des individus - ici du «criminel»- et inscrivant celle-ci dans une échelle de dignité des êtres ${ }^{33}$.

Ces nouvelles conditions ont enfin des incidences sur les liens entre les modalités de la reconnaissance et de la connaissance d'autrui. Au tout début du dix-neuvième siècle, le réseau des liens personnels est si serré, si constitutif des rapports sociaux, qu'il autorise l'usage des mêmes procédés pour reconnaître l'individu et connaître son - ou ses appartenance(s) collective(s). En effet, la classe d'individus à laquelle on appartient recoupe encore d'assez près les cellules locales ou professionnelles de rapports interpersonnels. L'identité générique de l'individu, le type social auquel il appartient, sont comme fondus dans le tissu serré de ce réseau de relations personnalisées. Dans cette société où prévaut, comme on l'a souligné supra, la "logique du face-à-face " (Piazza, 2004, p. 26), où le visage familier est la norme, l'identification de l'appartenance sociale de l'individu - son nom commun - et la détermination de son 
identité individuelle - son nom propre - s'effectuent dans le même mouvement. Plus exactement, les deux procédures sont indissociables, la connaissance du type social auquel on appartient s'effectuant indistinctement à travers la perception des traits de l'identité individuelle, et réciproquement. Or, pour les raisons évoquées précédemment, sous la Troisième République, les conditions qui rendent possible cette détermination simultanée ne sont plus réunies. En conséquence, dans une telle société, la connaissance et la reconnaissance ne peuvent s'effectuer que par des procédures disjointes, bien spécifiques. Elles deviennent alors le propre d'institutions dont les tâches respectives s'en trouvent davantage définies et spécialisées. Comme l'illustrent les différences entre le raisonnement des anthropologues et celui de Bertillon, l'identité générique doit être établie par des procédés bien distincts de ceux qui sont utilisés pour l'identification des individus, elle-même considérablement brouillée. La première voie sera explorée, plus spécifiquement, par les sciences humaines ou les organes statistiques de l'État ${ }^{34}$, la seconde par la police et l'administration ${ }^{35}$.

\section{Conclusion} celles de Bertillon comme des discours qui s'inscrivent non pas seulement dans un registre causal et étiologique, mais également dans un registre indiciaire et sémiologique. Souligner la fécondité de cette approche n'implique pas, bien entendu, que l'on doive minorer l'importance des aspects étiologiques de ces discours. Ces aspects ont une incidence et un relief tout particuliers lorsque ces acteurs abordent la question du traitement du crime. Toutefois, les savants dont il est ici question cherchent également, et peut-être avant tout, à mettre au jour les signes corporels indiquant l'appartenance au type criminel. De manière significative, Lombroso, par exemple, ne s'interroge guère sur la façon dont les caractéristiques physiques qu'il repère chez un pourcentage, selon lui, significatif de criminels - par exemple la "fossette occipitale» (Lombroso, 1871) - entraîneraient, comme une conséquence physiologique, un comportement condamné par le droit. Il prend acte d'une corrélation statistique qu'il observe et qui permet, selon lui, d'établir les indices de la disposition criminelle. Et il paraît souvent trouver là un enseignement qui se suffit à lui-même.

Sous l'éclairage que nous proposons ici, ce qui paraît commun à ces perspectives sur le crime, y compris celle de Bertillon, c'est donc le thème du corps comme lieu d'indication d'une identité - générique ou individuelle. Les discours produits par les différents acteurs sur ce thème - anthropologues, policiers, juristes, mais aussi écrivains -

Crime, Histoire \& Sociétés / Crime, History \& Societies, Vol. 12, $\mathrm{n}^{\circ} 1$ | 2008 
peuvent être alors regardés comme autant de théories du signe différentes, dont la logique répond à des objectifs, dans chaque cas, bien spécifiques. Ainsi, chez Bertillon, le signe - la partie du corps choisie - a-t-il une "puissance signalétique » (Bertillon, 1893). Ses différentes valeurs indiquent des différences individuelles et permettent d'identifier chaque individu en l'isolant dans le tableau continûment gradué de ressemblances et de dissemblances imperceptibles que la foule offre au regard du policier. Chez les naturalistes et les anthropologues qui s'appuient sur le principe de corrélation des formes organiques, il a une tout autre signification, qui le rapproche d'un sens classique : il a une puissance d'indexation ou d'indication, au sens de Charles S. Pierce ${ }^{36}$.

52 La perspective proposée ici permet également de faire ressortir les lacunes de l'explication de l'émergence de ces discours sur le crime par l'«air du temps ", en particulier l'«air du temps biologisant». Comme on a tenté de le montrer, une telle explication ne permet pas de rendre compte avec précision de la signification des modes d'appropriation de la biologie et, surtout, des différences qu'ils présentent selon les types d'acteur : ici le savant et le policier.

Enfin, les éléments livrés par cette perspective ouvrent sur une question plus générale, que l'on laissera ici largement ouverte. L'objectif du présent article était de souligner certaines différences entre les usages de l'histoire naturelle au dix-neuvième siècle à propos du crime, ainsi que le rôle des conditions historiques propres à la France des débuts de la Troisième République - le brouillage de l'identité - dans l'émergence et le succès de ces discours et pratiques. Une telle hypothèse implique, comme on l'a évoqué en introduction, que ces références à la biologie ont de fortes spécificités à la fois conceptuelles et contextuelles. Or ceci invite, nous semble-t-il, à considérer avec prudence les rapprochements trop rapides susceptibles d'être effectués entre la criminologie naturaliste du dix-neuvième siècle, d'une part, et les discours sur le crime qui invoquent aujourd'hui les sciences de la vie, d'autre part. Ce n'est pas à dire, bien entendu, qu'il n'y a pas de filiations entre les seconds et la première ; encore moins que ceux-là seraient plus « solides » ou davantage "scientifiquement fondés » que celle-ci : le présent article n'entend pas se situer dans ce type de registre évaluatif. Simplement, on peut se demander si l'on ne risque pas de succomber à une forme de présentisme lorsque l'on rapproche, sans plus de précisions, sous des dénominations unitaires " discours biologisants", «naturalisants", « essentialistes", « sociobiologiques", "héréditaristes", etc.-, comme s'ils procédaient d'une même logique interne et externe, les vues, par exemple, d'un Lombroso, et celles de la sociobiologie du crime, ou encore des recherches neurophysiologiques actuelles sur les mécanismes biochimiques susceptibles d'être impliqués dans l'agressivité. Même s'il existe assurément des liens entre ces discours d'un siècle à l'autre, même si des idéologies semblables peuvent venir s'y loger - en particulier les idéologies liées aux normes dominantes - l'historien doit également tenir compte du fait que les modèles biologiques de référence ${ }^{37}$, tout comme les contextes, diffèrent sous des aspects qui peuvent être essentiels. Aussi est-il sans doute important, dans la perspective d'une histoire comparative des idées, d'étudier dans le détail les éventuelles différences que présentent tous ces discours qui s'inspirent de la biologie au sens large. C'est là une des conditions préalables pour pouvoir estimer la réalité et la force du lien généalogique susceptible de les rattacher les uns aux autres, pour savoir si les discours contemporains sont des résurgences du passé ou s'ils ont des spécificités qui les en éloignent. 


\section{BIBLIOGRAPHIE}

About, I., Couleurs des yeux, lignes de la main, courbes du nez. De l'examen des corps à l'identification du criminel à la fin du XIX ${ }^{\mathrm{e}}$ siècle, communication au Colloque Récidive et récidivistes : de la Renaissance au XX $X^{e}$ siècle, organisé par Clive Emsley et Michel Porret, Université de Genève, 6-8 juin 2002, Résumé des communications, http://www.unige.ch/lettres/istge/ colreci.html, p. 7.

About, I., Les fondations d'un système national d'identification policière en France (1893-1914). Anthropométrie, signalements et fichiers, Genèses, 2004, 54, pp. 28-52.

Balan, B., L'ordre et le temps. L'anatomie comparée et l'histoire des vivants au XIXe siècle, Paris, Vrin, 1979.

Balzac, H. de, Avant-propos à La Comédie humaine, in Balzac, H. de, La Comédie humaine, Paris, Gallimard, coll. « Bibliothèque de la Pléiade », 1976 [1842], 1, pp. 7-20.

Berlière, J.-M., Le préfet Lépine. Vers la naissance de la police moderne, Paris, Denoël, 1993.

Berlière, J.-M., Le monde des polices en France, Bruxelles, Éd. Complexe, 1996.

Bernardini, J.-M., Le darwinisme social en France (1859-1918). Fascination et rejet d'une idéologie, Paris, Éditions du CNRS, 1997.

Bertillon, A., Identification anthropométrique. Instructions signalétiques, Melun, Imprimerie administrative, 1893.

Blanckaert, C., L'anthropologie en France, le mot et l'histoire, Bulletins et mémoires de la Société d'Anthropologie de Paris, 1989, n.s., I, 3-4, pp. 13-44.

Blanckaert, C., Des sauvages en pays civilisé. L'anthropologie des criminels (1850-1900), in Mucchielli, L. (dir.), Histoire de la criminologie française, Paris, L'Harmattan, 1994, pp. 55-88.

Briegel, F., Porret, M. (dir.), Le criminel endurci. Récidive et récidivistes du Moyen-Âge au XXe siècle, Genève, Droz, 2006.

Coffin, J.-C., Le corps social en accusation. Le thème de la dégénérescence en France et en Italie. 1850-1900, Paris, Thèse de l'Université Paris VII, 1993.

Darmon, P., Présentation de Lombroso, C., La femme criminelle et la prostituée, Grenoble, Jérôme Millon, coll. « Mémoires du corps », 1991.

Daudin, H., De Linné à Lamarck. Méthodes de la classification et idée de série en botanique et en zoologie (1740-1790), Paris, Félix Alcan, 1926-1927 (réimprimé par les Éditions des Archives Contemporaines en 1983).

Desrosières, A., La politique des grands nombres. Histoire de la raison statistique, Paris, La Découverte, 1993.

Durkheim, É., De la division du travail social, Paris, Félix Alcan, 1893.

Foucault, M., Les mots et les choses, Paris, Gallimard, 1966.

Ginzburg, C., Signes, traces, pistes. Racines d'un paradigme de l'indice, Le Débat, novembre 1980, 6, pp. 3-45.

Guillo, D., Les figures de l'organisation. Sciences de la vie et sciences sociales au XIX ${ }^{e}$ siècle, Paris, PUF, 2003. 
Guillo, D., À la recherche des signes de l'identité. Balzac et l'histoire naturelle, Politix, 2006, 74, pp. 49-74.

Kaluszynski M., Alphonse Bertillon et l'anthropométrie, in Faure, A., Vigier, P. (dir.), Maintien de l'ordre et police en France au XIXe siècle, 1880-1920, Paris, Créaphis, 1987, pp. 269-286.

Kaluszynski, M., La République à l'épreuve du crime. La construction du crime comme objet politique, Paris, LGDJ, 2002.

L'Heuillet, H., Basse politique, haute police. Une approche historique et philosophique de la police, Paris, Fayard, 2001.

Lamarck, J.-B. de, Flore française ou description succincte de toutes les plantes qui croissent en France, Paris, Imprimerie Royale, 1778, t. 1, « Discours préliminaire ».

Lauvergne, H., Les forçats considérés sous le rapport physiologique, moral et intellectuel, observés au bagne de Toulon, publié sous le titre Les forçats, Grenoble, Jérôme Millon, coll. « Mémoires du corps », 1991 [1841].

Lombroso, C., Esistenza di una fossa occipitale mediana nel cranio di un delinquente, Rendiconto del R. Istituto Lombardo di Scienze e Lettere, 1871, $2^{\mathrm{e}}$ série, IV, pp. 37-41.

Lombroso, C., L'uomo delinquente studiato in rapporto alla antropologia, alla medicina legale e alle discipline carcerarie, Milan, Hoepli, 1876.

Lombroso, C., Ferrero, G., La femme criminelle et la prostituée, Paris, Félix Alcan, 1896.

Mucchielli, L. (dir.), Histoire de la criminologie française, Paris, L'Harmattan, 1994.

Mucchielli, L., La découverte du social. Naissance de la sociologie en France (1870-1914), Paris, La Découverte, 1998.

Noiriel, G., Réfugiés et sans-papiers. La République face au droit d'asile. XIX ${ }^{e}-X X^{e}$ siècles, Paris, CalmannLévy, 1991.

Noiriel, G., Les origines républicaines de Vichy, Paris, Hachette Littératures, coll. « Histoires », 1999.

Piazza, P., Histoire de la carte nationale d’identité, Paris, Odile Jacob, 2004.

Pick, D., Faces of Degeneration. A European Disorder, c.1848-c.1918, Cambridge, Cambridge University Press, 1989.

Pierce, C. S., Écrits sur le signe, traduits et commentés par G. Deledalle, Paris, Seuil, 1978.

Renneville, M., La médecine du crime. Essai sur l'émergence d'un regard médical sur la criminalité en France (1785-1885), Lille, Presses Universitaires du Septentrion, 1997.

Schnapper, B., La récidive, une obsession créatrice au XIX ${ }^{\mathrm{e}}$ siècle, in Collectif, $\mathrm{XXI}^{e}$ congrès de l'Association française de criminologie : le récidivisme, Paris, PUF, 1983, pp. 25-64.

\section{NOTES}

2. Lorsqu'ils font référence au corps du «criminel» ou aux sciences de la vie de leur temps, les acteurs dont il est ici question reprennent pour l'essentiel les concepts et les propositions mobilisés dans le cadre de la médecine et de l'histoire naturelle. Durant les deux premiers tiers du XIX ${ }^{\mathrm{e}}$ siècle, l'histoire naturelle, appuyée sur l'anatomie comparée, constitue globalement la discipline dominante dans les réflexions savantes sur le vivant, avant que la physiologie expérimentale ne la supplante progressivement durant le dernier tiers du siècle (voir Balan, 
1979). Elle reste, toutefois, encore dominante dans les dernières décennies du siècle dans les parties les plus spéculatives des sciences de la vie ou dans certaines disciplines comme l'anthropologie, qui prend directement son essor à partir des réflexions des médecins et des naturalistes dès le milieu du siècle (voir infra; Blanckaert, 1989).

3. Pour des exemples d'analyses de ce type, voir, par exemple, certains articles consacrés à ce thème dans Mucchielli (1994).

4. On trouve une telle suggestion de lecture dans Ginzburg (1980), et dans Noiriel (1991,pp. 156-170). On notera, sur ce point, que la nature des sous-disciplines des sciences de la vie auxquelles empruntent les discours évoqués ici, attire d'emblée l'attention en direction de l'hypothèse formulée dans le présent article, c'est-à-dire l'hypothèse d'un registre indiciaire. La médecine, avec son versant diagnostique, voué à l'identification des signes de la maladie, et l'histoire naturelle, dont le but est de constituer des groupes d'êtres vivants sur la base de «caractères indicateurs" susceptibles de servir d'indices à des ressemblances spécifiques fondamentales, constituent autant de savoirs qui relèvent sous des aspects essentiels d'un « paradigme de l'indice » (Ginzburg, 1980).

5. Sur Alphonse Bertillon et le problème de l'identité, voir Ginzburg (1980) ; Kaluszynski (1987, 2002) ; Berlière (1996, pp. 41-68) ; Noiriel (1999, pp. 188-204); Piazza (2004, pp. 77-122).

6. Selon l'expression de Pierre Piazza (2004, p. 26). Nous évoquons ce point, également, et un peu plus abondamment que nous ne le faisons ici, dans Guillo (2006).

7. La législation sur les criminels récidivistes a eu d'importantes conséquences sur le développement des systèmes d'identification et a entraîné dans son sillage ou accompagné d'amples réorganisations administratives - en particulier la création d'un service de l'identité judiciaire en 1893 - comme le montre de façon très précise et détaillée Ilsen About (About, 2004, en particulier pp. 30-31).

8. Sur ce point, voir Berlière (1993, par exemple, p. 225).

9. Bertillon, par exemple, évoque ce fait dans Bertillon (1893, p. XIV). Il indique également que « les nouvelles lois pénales, dites de relégation, de libération conditionnelle et de sursis de peines (Lois Bérenger), ont singulièrement augmenté le nombre des dissimulations d'identité par cela même qu'elles les rendaient plus tentantes » (Bertillon, 1893, pp. XIII-XIV).

10. Bien entendu, la police utilise également des méthodes non administratives, dont certaines s'avèrent efficaces : dénonciations, "moutons " dans les cellules, chargés de recueillir les confidences, etc. Sur ces points, voir Berlière (1993, par exemple, p. 226).

11. Le service photographique de la Préfecture de police de Paris est créé et organisé en 1874 par le préfet Léon Renault.

12. Sur les traces, les marques et les empreintes, comme moyen d'identification individuelle utilisé par la police, voir L'Heuillet (2001, pp. 294-312).

13. Sur les réserves formulées initialement à propos de l'efficacité des empreintes digitales et, plus généralement, de tous les types de procédure par "empreinte», voir Bertillon (1893, pp. XVI-XVI). La dactyloscopie s'imposera, toutefois, par la suite : voir, par exemple, L'Heuillet (2001, p. 295).

14. Sur Bertillon et la constitution du service de l'identité judiciaire à Paris en 1893, on se reportera à Kaluszynski $(1987,2002)$; sur les «adaptations de l'administration policière " qui accompagnent le processus de « constitution d'un système d'identification et de recherches » des criminels en France de 1893 à 1914, voir About (2004).

15. On notera que l'anthropométrie ne constitue qu'une des techniques proposées par Bertillon pour l'identification des criminels. Ce que l'on nomme le «bertillonnage » comprend également le «portrait parlé », la photographie signalétique, le relevé des marques corporelles ou encore l'analyse graphologique. Sur ces points, voir Kaluszynski $(1987,2002)$; L'Heuillet (2001) ; About (2004).

16. Citation tirée de Berlière (1993, p. 228). 
17. Sur ce point, voir L'Heuillet (2001, p. 294), qui relève l'importance de cette phrase.

18. Voir également, Bertillon (1893, p. III) : « Le surveillant de prison qui passe son existence en tête à tête avec des êtres humains et qui, à proprement parler, est un gardien d'hommes doit tendre à devenir un anthropologiste ».

19. Les " recherches doubles ", c'est-à-dire dans deux fichiers connexes, s'imposent lorsqu'une mesure effectuée sur un suspect est proche d'une valeur séparant deux classes.

20. Aussi l'idée selon laquelle la méthode anthropométrique s'accompagnerait «de la détermination d'une figure moyenne légitimée par l'outil statistique qui rend tangible la définition de stigmates criminels dont l'anthropologie criminelle, à la même époque, et malgré les débats internes qui la traversent, fait son centre d'intérêt » (About, 2002, résumé) nous paraitelle pouvoir être discutée.

21. L'histoire naturelle, rappelons-le, constitue la discipline dominante dans les sciences de la vie durant une bonne partie du siècle.

22. Voir, en particulier, Lombroso, Ferrero (1896).

23. Voir, en particulier, Lauvergne (1991 [1841]), et Lombroso (1876).

24. On retrouve ce thème jusque chez Émile Durkheim, dans les références que celui-ci fait à l'anthropologie de son temps, notamment dans Durkheim (1893). Sur les liens conceptuels entre l'histoire naturelle et cette anthropologie, voir Blanckaert (1989); Bernardini (1997); Guillo (2003, troisième partie, Chapitre 1).

25. Sur ces points, voir, en particulier, Darmon (1991) ; Blanckaert (1994).

26. L'importance de ce texte, pour les débats agités autour de cette question, a été soulignée, en particulier, par Michel Foucault (1966, p. 243).

27. Cette distinction nette entre le travail du savant et celui du policier rend possible le développement d'une forme de savoirs et de pratiques à l'interface entre ces deux activités. En cette fin de XIXe siècle, un nouvel acteur prend ainsi une importance croissante: l'expert judiciaire, qui développe une technique, un savoir appliqué, destiné à servir à un pouvoir de contrôle, différent d'un savoir fondamental, affichant un objectif de connaissance, comme celui dont se réclame l'anthropologie criminelle.

28. Avec, bien entendu, un objectif tout autre, et probablement sans en avoir conscience, du moins sans s'en soucier.

29. En ce sens, la méthode d'identification anthropométrique proposée par Bertillon repose sur un principe « d'identification négative », comme le souligne Hélène L'Heuillet (2001, p. 306).

30. Sur tous ces éléments, voir Piazza (2004), première partie, chapitres I et II.

31. Sur tous ces éléments, voir ibid.

32. Nous avons tenté de développer ce point à propos de Balzac in Guillo (2006).

33. C'est ainsi l'usage qu'en font les criminologues naturalistes, mais aussi, avant eux, Balzac. L'Avant-propos à la Comédie humaine place ainsi l'ouvrage explicitement dans le prolongement des travaux de Cuvier et Geoffroy Saint-Hilaire : voir Balzac (1976 [1842] pp. 8-9). Sur ces points, voir Guillo (2006).

34. Sur ce point, voir Desrosières (1993).

35. Tous les points évoqués dans cette section sont développés avec davantage de détails et de précisions in Guillo (2006).

36. Chez Pierce, l'« index» ou « indice » est la conséquence d'une connexion physique entre le signe et son référent. Sur la définition de l'«index » et la distinction "symbole ", "index », « icône », voir Pierce (1978, pp. 147-160).

37. Au plan des concepts, la génétique et la neurophysiologie actuelles dessinent des cadres de pensée qui n'ont guère de rapport avec l'anatomie comparée et la théorie de l'hérédité du dixneuvième siècle, sur lesquelles s'appuient les criminologues évoqués ici. 


\section{RÉSUMÉS}

La référence à l'histoire naturelle dans les discours sur le crime de la fin du dix-neuvième siècle ne procède pas seulement de préoccupations d'ordre étiologique, visant à démontrer le fondement organique et héréditaire du crime. Elle se déploie également dans le registre des indices de l'identité, individuelle et collective. Envisagés sous cet angle, ces discours apparaissent comme des constructions intellectuelles répondant au problème du brouillage de l'identité, qui marque les dernières décennies du siècle. En outre, cette référence à l'histoire naturelle prend des formes très différentes dans le monde savant, d'une part - l'anthropologie criminelle -, et dans le monde judiciaire, d'autre part - en particulier chez Bertillon. Dans chacun des cas, elle est mise au service de besoins spécifiques: d'un côté, la connaissance de l'identité générique de l'individu; de l'autre, la reconnaissance de l'identité individuelle.

The reference to natural history in discourse on crime at the end of the nineteenth Century is not based solely on an etiological perspective which would aim at demonstrating that crime is of organic and hereditary nature. Such a reference is also present with respect to individual and collective identity. From this perspective, these discourses represent intellectual constructions which address the issue of blurred identities, an important issue in the final decades of the nineteenth century. Moreover, this reference to natural history takes very different forms, depending on whether used in the academic sphere - esp. criminal anthropology - or in the judicial sphere, esp. by Bertillon. In each of these cases, it is subjected to specific needs : on one hand, knowledge of the generic identity of the individual ; and, on the other hand, recognition of individual identity.

\section{AUTEUR}

\section{DOMINIQUE GUILLO}

GEMAS (UMR 8598, Maison des Sciences de l'Homme, 54, boulevard Raspail, 75006 Paris, dominiqueguillo@yahoo.fr Dominique Guillo est chargé de recherche au CNRS. Ses principales publications : À la recherche des signes de l'identité : Balzac et l'histoire naturelle, Politix (2006, 74, pp. 49-74); Les figures de l'organisation. Sciences de la vie et sciences sociales au XIXe siècle, Paris, PUF, 2003 ; La sociologie d'inspiration biologique au dix-neuvième siècle : une science de l'« organisation » sociale, Revue française de sociologie (2 000, pp. 241-275). Il travaille actuellement sur les usages de la biologie dans les réflexions sur l'identité au XIX ${ }^{e}$ siècle, les représentations non-savantes de l'évolution de l'homme et du darwinisme aujourd'hui, les relations êtres humains/chiens. 